\title{
Informative Laplacian Projection
}

\author{
Zhirong Yang and Jorma Laaksonen \\ Department of Information and Computer Science ${ }^{\star}$ \\ Helsinki University of Technology \\ P.O. Box 5400, FI-02015, TKK, Espoo, Finland \\ \{zhirong. yang, jorma. laaksonen\}@tkk.fi
}

\begin{abstract}
A new approach of constructing the similarity matrix for eigendecomposition on graph Laplacians is proposed. We first connect the Locality Preserving Projection method to probability density derivatives, which are then replaced by informative score vectors. This change yields a normalization factor and increases the contribution of the data pairs in low-density regions. The proposed method can be applied to both unsupervised and supervised learning. Empirical study on facial images is provided. The experiment results demonstrate that our method is advantageous for discovering statistical patterns in sparse data areas.
\end{abstract}

\section{Introduction}

In image compression and feature extraction, linear expansions are commonly used. An image is projected on the eigenvectors of a certain positive semidefinite matrix, each of which provides one linear feature. One of the classical approaches is the Principal Component Analysis (PCA), where the variance of images in the projected space is maximized. However, the projection found by PCA may not always encode locality information properly.

Recently, many dimensionality reduction algorithms using eigendecomposition on a graph-derived matrix have been proposed to address this problem. This stream of research has been stimulated by the methods Isomap [1] and Local Linear Embedding [2, which have later been unified as special cases of the Laplacian Eigenmap [3. The latter minimizes the local variance while maximizing the weighted global variance. The Laplacian Eigenmap has also shown to be a good approximation of both the Laplace-Beltrami operator for a Riemannian manifold [3] and the Normalized Cut for finding data clusters [4]. A linear version of the Laplacian Eigenmap algorithm, the Locality Preserving Projection (LPP) 5, as well as many other locality-sensitive transformation methods such as the Hessian Eigenmap [6] and the Local Tangent Space Alignment 7], have also been developed.

However, little research effort has been devoted to graph construction. Locality in the above methods is commonly defined as a spherical neighborhood

\footnotetext{
* Supported by the Academy of Finland in the project Finnish Centre of Excellence in Adaptive Informatics Research.
} 
around a vertex (e.g. [18]). Two data points are linked with a large weight if and only if they are close, regardless of their relationship to other points. A Laplacian Eigenmap based on such a graph tends to overly emphasize the data pairs in dense areas and is therefore unable to discover the patterns in sparse areas. A widely used alternative to define the locality (e.g. 119]) is by $k$-nearest neighbors $(k-\mathrm{NN}, k \geq 1)$. Such definition however assumes that relations in each neighborhood are uniform, which may not hold for most real-world data analysis problems. Combination of a spherical neighborhood and the $k$-NN threshold has also been used (e.g. [5]), but how to choose a suitable $k$ remains unknown. In addition, it is difficult to connect the $k$-NN locality to the probability theory.

Sparse patterns, which refer to the rare but characteristic properties of samples, play essential roles in pattern recognition. For example, moles or scars often help people identify a person by appearance. Therefore, facial images with such features should be more precious than those with an average face when for example training a face recognition system. A good dimensionality reduction method ought to make the most use of the former kind of samples while associating relatively low weights to the latter.

We propose a new approach to construct a graph similarity matrix. First we express the LPP objective in terms of Parzen estimation, after which the derivatives of the density function with respect to difference vectors are replaced by the informative score vectors. The proposed normalization principle penalizes the data pairs in dense areas and thus helps discover useful patterns in sparse areas for exploratory analysis. The proposed Informative Laplacian Projection (ILP) method can then reuse the LPP optimization algorithm. ILP can be further adapted to the supervised case with predictive densities. Moreover, empirical results of the proposed method on facial images are provided for both unsupervised and supervised learning tasks.

The remaining of the paper is organized as follows. The next section briefly reviews the Laplacian Eigenmap and its linear version. In Section 3 we connect LPP to the probability theory and present the Informative Laplacian Projection method. The supervised version of ILP is described in Section 4. Section 5 provides the experiment results on unsupervised and supervised learning. Conclusions as well as future work is finally discussed in Section 6.

\section{Laplacian Eigenmap}

Given a collection of zero-mean samples $\mathbf{x}^{(i)} \in \mathbb{R}^{M}, i=1, \ldots, N$, the Laplacian Eigenmap 3] computes an implicit mapping $f: \mathbb{R}^{M} \mapsto \mathbb{R}$ such that $y^{(i)}=$ $f\left(\mathbf{x}^{(i)}\right)$. The mapped result $\mathbf{y}=\left[y^{(1)}, \ldots, y^{(N)}\right]^{T}$ minimizes

$$
\mathcal{J}(\mathbf{y})=\sum_{i=1}^{N} \sum_{j=1}^{N} S_{i j}\left(y^{(i)}-y^{(j)}\right)^{2}
$$

subject to $\mathbf{y}^{T} \mathbf{D} \mathbf{y}=1$, where $\mathbf{S}$ is a similarity matrix and $\mathbf{D}$ a diagonal matrix with $D_{i i}=\sum_{j=1}^{N} S_{i j}$. A popular choice of $\mathbf{S}$ is the radial Gaussian kernel: 


$$
S_{i j}=\exp \left(-\frac{\left\|\mathbf{x}^{(i)}-\mathbf{x}^{(j)}\right\|^{2}}{2 \sigma^{2}}\right),
$$

with a positive kernel parameter $\sigma .\left\{S_{i j}\right\}_{i, j=1}^{N}$ can also be regarded as the edge weights of a graph where the data points serve as vertices.

The solution of the Laplacian Eigenmap (1) can be found by solving the generalized eigenproblem

$$
(\mathbf{D}-\mathbf{S}) \mathbf{y}=\lambda \mathbf{D y} .
$$

An $R$-dimensional $(R \ll M)$ compact representation of the data set is then given by the eigenvectors associated with the second least to $(R+1)$-th least eigenvalues.

The Laplacian Eigenmap outputs only the transformed results of the training data points without an explicit mapping function. One has to rerun the whole algorithm for newly coming data. This drawback can be overcome by using parameterized transformations, among which the simplest way is to restrict the mapping to be linear: $y=\mathbf{w}^{T} \mathbf{x}$ for any input vector $\mathbf{x}$ with $\mathbf{w} \in \mathbb{R}^{M}$. Let $\mathbf{X}=$ $\left[\mathbf{x}^{(1)}, \ldots, \mathbf{x}^{(N)}\right]$. The linearization leads to the Locality Preserving Projection (LPP) [5] whose optimization problem is

$$
\begin{aligned}
\operatorname{minimize} & \mathcal{J}_{\mathrm{LPP}}(\mathbf{w})=\mathbf{w}^{T} \mathbf{X}(\mathbf{D}-\mathbf{S}) \mathbf{X}^{T} \mathbf{w} \\
\text { subject to } & \mathbf{w}^{T} \mathbf{X} \mathbf{D X}^{T} \mathbf{w}=1
\end{aligned}
$$

with the corresponding eigenvalue solution:

$$
\mathbf{X}(\mathbf{D}-\mathbf{S}) \mathbf{X}^{T} \mathbf{w}=\lambda \mathbf{X} \mathbf{D} \mathbf{X}^{T} \mathbf{w} .
$$

Then the eigenvectors with the second least to $(R+1)$-th least eigenvalues form the columns of the $R$-dimensional transformation matrix $\mathbf{W}$.

\section{Informative Laplacian Projection}

With the radial Gaussian kernel, the Laplacian Eigenmap or LPP objective (11) weights the data pairs only according to their distance without considering the relationship between their vertices and other data points. Moreover, it is not difficult to see that the $\mathbf{D}$ matrix actually measures the "importance" of data points by their densities, which overly emphasizes some almost identical samples. Consequently, the Laplacian Eigenmap and LPP might fail to preserve the statistical information of the manifold in some sparse areas even though a vast amount of training samples were available. Instead, they could encode some tiny details which are difficult to interpret (see e.g. Fig. 4 in [5] and Fig. 2 in 10 ).

To attack this problem, let us first rewrite the objective (1) with the density estimation theory: 


$$
\begin{aligned}
\mathcal{J}_{\text {LPP }}(\mathbf{w}) & =\mathbf{w}^{T}\left[\sum_{i=1}^{N} \sum_{j=1}^{N} S_{i j}\left(\mathbf{x}^{(i)}-\mathbf{x}^{(j)}\right)\left(\mathbf{x}^{(i)}-\mathbf{x}^{(j)}\right)^{T}\right] \mathbf{w} \\
& =-\mathbf{w}^{T}\left[\sum_{i=1}^{N} \sum_{j=1}^{N} \frac{N}{2 \sigma^{2}} \frac{\partial\left(\frac{1}{N} \sum_{k=1}^{N} S_{i k}\right)}{\partial\left(\mathbf{x}^{(i)}-\mathbf{x}^{(j)}\right)}\left(\mathbf{x}^{(i)}-\mathbf{x}^{(j)}\right)^{T}\right] \mathbf{w} \\
& =\text { const } \cdot \mathbf{w}^{T}\left[\sum_{i=1}^{N} \sum_{j=1}^{N} \frac{\partial \hat{p}\left(\mathbf{x}^{(i)}\right)}{\partial \mathbf{\Delta}^{(i j)}}\left(\boldsymbol{\Delta}^{(i j)}\right)^{T}\right] \mathbf{w},
\end{aligned}
$$

where $\boldsymbol{\Delta}^{(i j)}$ denotes $\mathbf{x}^{(i)}-\mathbf{x}^{(j)}$ and $\hat{p}\left(\mathbf{x}^{(i)}\right)=\sum_{k=1}^{N} S_{i k} / N$ is recognized as a Parzen window estimation of $p\left(\mathbf{x}^{(i)}\right)$.

Next, we propose the Informative Laplacian Projection (ILP) method by using the information function $\log \hat{p}$ instead of raw densities $\hat{p}$ :

$$
\operatorname{minimize} \mathcal{J}_{\mathrm{ILP}}(\mathbf{w})=-\mathbf{w}^{T}\left[\sum_{i=1}^{N} \sum_{j=1}^{N} \frac{\partial \log \hat{p}\left(\mathbf{x}^{(i)}\right)}{\partial \boldsymbol{\Delta}^{(i j)}}\left(\boldsymbol{\Delta}^{(i j)}\right)^{T}\right] \mathbf{w}
$$

subject to $\mathbf{w}^{T} \mathbf{X} \mathbf{X}^{T} \mathbf{w}=1$.

The use of the log function arises from the fact that partial derivatives on the log-density can yield a normalization factor:

$$
\begin{aligned}
\mathcal{J}_{\text {ILP }}(\mathbf{w}) & =\mathbf{w}^{T}\left[\sum_{i=1}^{N} \sum_{j=1}^{N} \frac{S_{i j}}{\sum_{k=1}^{N} S_{i k}} \boldsymbol{\Delta}^{(i j)}\left(\boldsymbol{\Delta}^{(i j)}\right)^{T}\right] \mathbf{w} \\
& =\sum_{i=1}^{N} \sum_{j=1}^{N} E_{i j}\left(y^{(i)}-y^{(j)}\right)^{2},
\end{aligned}
$$

where $E_{i j}=S_{i j} / \sum_{k=1}^{N} S_{i k}$. We can then employ the symmetrized version $\mathbf{G}=$ $\left(\mathbf{E}+\mathbf{E}^{T}\right) / 2$ to replace $\mathbf{S}$ in (6) and reuse the optimization algorithm of LPP except that the weighting in the constraint of LPP is omitted, i.e. $\mathbf{D}=\mathbf{I}$, because such weighting excessively stresses the samples in dense areas.

The projection found by our method is also locality preserving. Actually the ILP is identical to LPP for the manifolds such as the "Swiss roll" 112 or Smanifold [11] where the data points are uniformly distributed. However, ILP behaves very differently from LPP otherwise. The above normalization, as well as omitting the sample weights, penalizes the pairs in dense regions while increases the contribution of those in areas of lower-density, which is conducive to discovering sparse patterns. 


\section{Supervised Informative Laplacian Projection}

The Informative Laplacian Projection can be extended to the supervised case where each sample $\mathbf{x}^{(i)}$ is associated with a class label $c_{i}$ in $\{1, \ldots, Q\}$. The discriminative version just replaces $\log p\left(\mathbf{x}^{(i)}\right)$ in (10) with $\log p\left(c_{i} \mid \mathbf{x}^{(i)}\right)$. The resulting Supervised Informative Laplacian Projection (SILP) minimizes

$$
\mathcal{J}_{\text {SILP }}(\mathbf{w})=-\mathbf{w}^{T}\left[\sum_{i=1}^{N} \sum_{j=1}^{N} \frac{\partial \log \hat{p}\left(c_{i} \mid \mathbf{x}^{(i)}\right)}{\partial \boldsymbol{\Delta}^{(i j)}}\left(\boldsymbol{\Delta}^{(i j)}\right)^{T}\right] \mathbf{w}
$$

subject to $\mathbf{w}^{T} \mathbf{X} \mathbf{X}^{T} \mathbf{w}=1$. According to the Bayes theorem, we can write out the partial derivative with Parzen density estimations:

$$
-\frac{\partial \log p\left(c_{i} \mid \mathbf{x}^{(i)}\right)}{\partial \boldsymbol{\Delta}^{(i j)}}=S_{i j} \cdot \frac{1}{\sum_{k=1}^{N} S_{i k}} \cdot\left(\phi_{i j} \frac{\sum_{k=1}^{N} S_{i k}}{\sum_{k=1}^{N} S_{i k} \phi_{i k}}-1\right) \boldsymbol{\Delta}^{(i j)},
$$

where $\phi_{i j}=1$ if $c_{i}=c_{j}$ and 0 otherwise. The optimization of SILP is analogous to the unsupervised algorithm except

$$
E_{i j}=S_{i j} \cdot \frac{1}{\sum_{k=1}^{N} S_{i k}} \cdot\left(\phi_{i j} \frac{\sum_{k=1}^{N} S_{i k}}{\sum_{k=1}^{N} S_{i k} \phi_{i k}}-1\right) .
$$

The first two factors in (16) are identical to the unsupervised case, favoring local pairs, but penalizing those in dense areas. The third factor in parentheses, denoted by $\rho_{i j}$, takes the class information into account. It approaches zero when $\phi_{i j}=1$ and the class label remains almost unchanged in the neighborhood of $\mathbf{x}^{(i)}$. This neglects pairs that are far apart from the classification boundary. For other equi-class pairs, $\rho_{i j}$ takes a positive value if different class labels are mixed in the neighborhood, i.e. the pair is near the classification boundary. In this case SILP minimizes the variance of their difference vectors, which reflects the idea of increasing class cohesion. Finally, $\rho_{i j}=-1$ if $\phi_{i j}=0$, i.e. the vertices belong to different classes. SILP actually maximizes the norm of such edges in the projected space. This results in dilation around the classification boundary in the projected space, which is desired for discriminative purposes.

Unlike the conventional Fisher's Linear Discriminant Analysis (LDA) 12, our method does not rely on the between-class scatter matrix, which is often of low-rank and restricts the number of discriminants. Instead, SILP can produce discriminative components as many as the dimensionality of the original data. The additional dimensions can be beneficial for classification accuracy, as will be shown in Section 5.2 .

\section{Experiments}

\subsection{Learning of Turning Angles of Facial Images}

This section demonstrates the application of ILP on facial images. We have used 2,662 facial images from the FERET collection [13, in which 2409 are of pose 


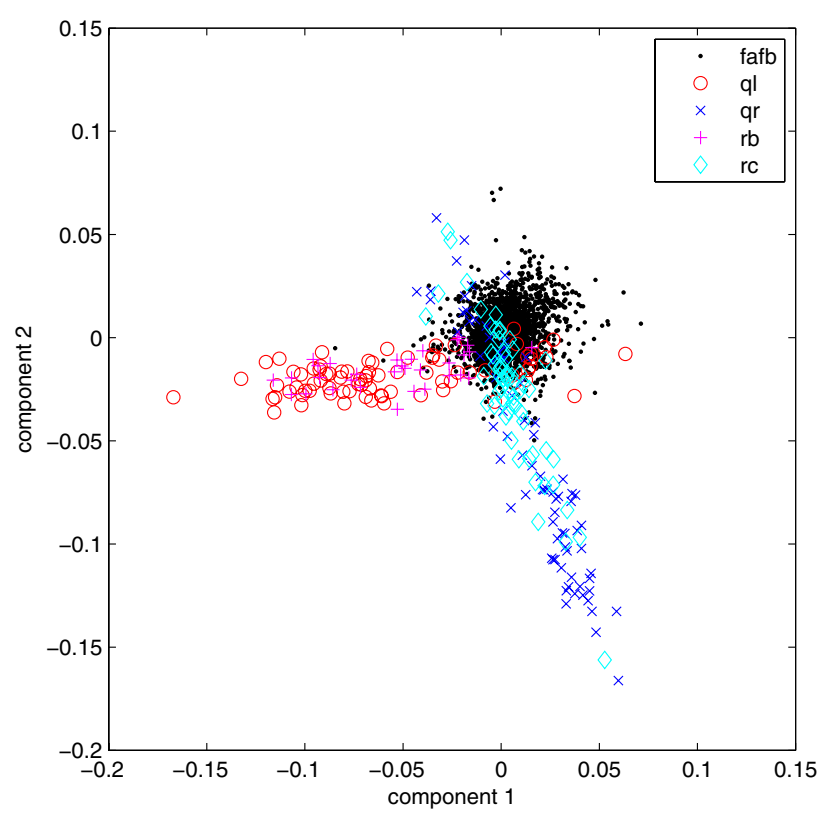

Fig. 1. FERET faces in the subspace found by ILP

$f a$ or $f b, 81$ of $q l, 78$ of $q r, 32$ of $r b$, and 62 of $r c$. The meanings of the FERET pose abbreviations are:

- fa: regular frontal image;

- $f b$ : alternative frontal image, taken shortly after the corresponding $f a$ image;

- ql: quarter left - head turned about 22.5 degrees left;

- qr: quarter right - head turned about 22.5 degrees right;

- rb: random image - head turned about 15 degree left;

- rc: random image - head turned about 15 degree right.

In summary, most images are of frontal pose except about 10 percent turning to the left or to the right. The unsupervised learning goal is to find the components that correspond to the left- and right-turning directions. In this work we obtained the coordinates of the eyes from the ground truth data of the collection. Afterwards, all face boxes were normalized to the size of $64 \times 64$, with fixed locations for the left eye $(53,17)$ and the right eye $(13,17)$.

We have tested three methods that use the eigenvalue decomposition on a graph: ILP (10)-(11), LPP (4)-(5), and the linearized Modularity [14 method. The original facial images were first preprocessed by Principal Component Analysis and reduced to feature vectors of 100 dimensions. The neighborhood parameter for the similarity matrix was empirically set to $\sigma=3.5$ in (2) for all the compared algorithms.

The data points in the subspace learned by ILP are shown in Figure 1 It can be seen that the faces with left-turning poses ( $q l$ and $r b$ ) mainly distribute along 

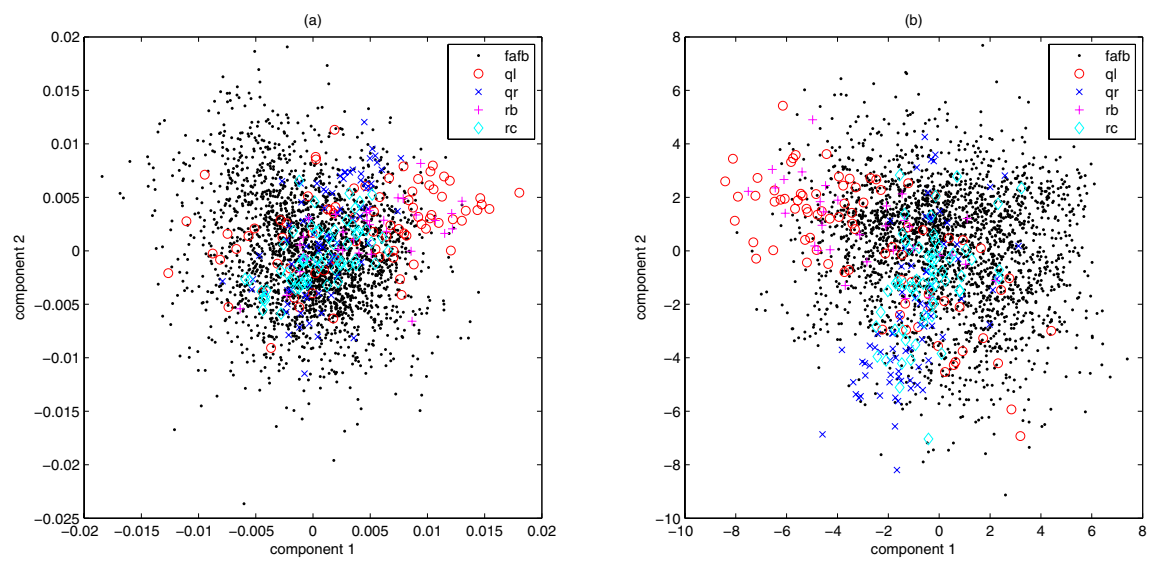

Fig. 2. FERET faces in the subspaces found by (a) LPP and (b) Modularity

the horizontal dimension while the right-turning faces ( $q r$ and $r c$ ) roughly along the vertical. The projected results of LPP and Modularity are shown in Figure2, As one can see, it is almost impossible to distinguish any direction related to a facial pose in the subspace learned by LPP. For the Modularity method, one can barely perceive the left-turning direction is associated with the horizontal dimension while the right-turning with the vertical. All in all, the faces with turning poses are heavily mixed with the frontal ones.

The resulting $\mathbf{W}$ contains three columns, each of which has the same dimensionality as the input feature vector and can thus be reconstructed to a filtering image via the inverse PCA transformation. If a transformation matrix works well for a given learning problem, it is expected to find some semantic connections between its filtering images and our common prior knowledge of the discrimination goal. The filtering images of ILP are displayed in the left-most column of Figure 3. from which one can easily connect the contrastive parts in these filtering images with the turning directions. The facial images on the right of the filtering images are the every sixth images with the least 55 projected values in the corresponding projected dimension.

\subsection{Discriminant Analysis on Eyeglasses}

Next we performed experiments for discriminative purposes on a larger facial image data set in the University of Notre Dame biometrics database distribution, collection B [15]. The preprocessing was similar to that for the FERET database. We segmented the inner part from 7,200 facial images, among which 2,601 are labeled as the subject in the image is wearing eyeglasses. We randomly selected 2,000 eyeglasses and 4,000 non-eyeglasses images for training and the rest for testing. The images of a same subject were assigned to either the training set or the testing set, never to both. The supervised learning task here is to analyze the discriminative components for recognizing eyeglasses. 


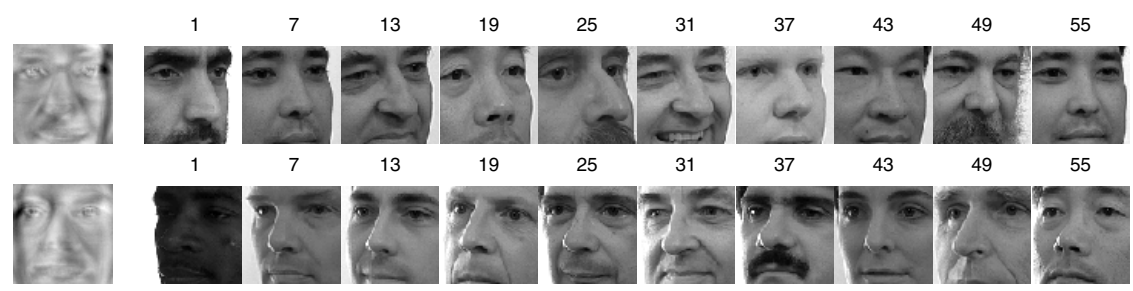

Fig. 3. The bases for turning angles found by ILP as well as the typical images with least values in the corresponding dimension. The top line is for the left-turning pose and the bottom for the right-turning. The numbers above the facial images are their ranks in the ascending order of the corresponding dimension.

(a)

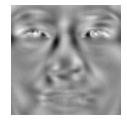

(c) (b)

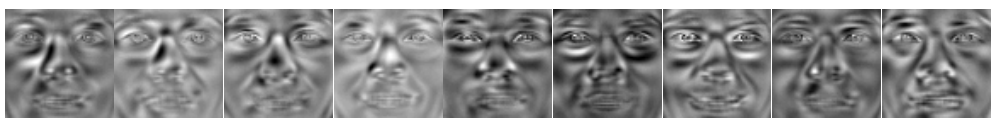

(d)
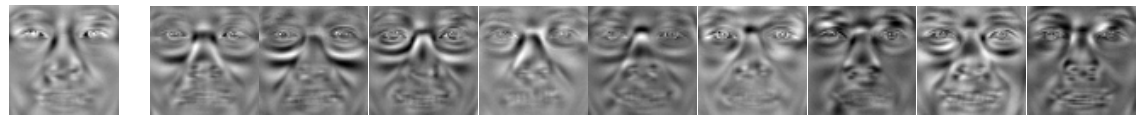

Fig. 4. Filtering images of four discriminant analysis methods: (a) LDA, (b) LSDA, (c) LSVM, and (d) SILP

We have compared four discriminant analysis methods: LDA [12], the Linear Support Vector Machine (LSVM) [16, the Locality Sensitive Discriminant Analysis (LSDA) 9], and SILP (14). The neighborhood width parameter $\sigma$ in (2) was empirically set to 300 for LSDA and SILP. The tradeoff parameters in LSVM and LSDA were determined by five-fold cross-validations. The filtering images learned by the above methods are displayed in Figure 4, LDA and LSVM can produce only one discriminative component for two-class problems. In this experiment, their resulting filtering images are very similar except some tiny differences, where the major effective filtering part appears in and between the eyes. The number of discriminants learned by LSDA or SILP is not restricted to one. One can see different contrastive parts in the filtering images of these two methods. In comparison, the top SILP filters are more Gabor-like and the wave packets are mostly related with the bottom rim of the glasses.

After transforming the data, we predicted the class label of each test sample by its nearest neighbor in the training set using the Euclidean distance. Figure [5] illustrates the classification error rates versus the number of discriminative components used. The performance of LDA and LSVM only depends on the first component, with classification error rates $16.98 \%$ and $15.51 \%$, respectively. Although the first discriminant of LSDA and SILP work not as well as the one of LDA, they both supersede LDA or even outperform LSVM with subsequent components added. With the first 11 projected dimensions, LSDA achieves its 


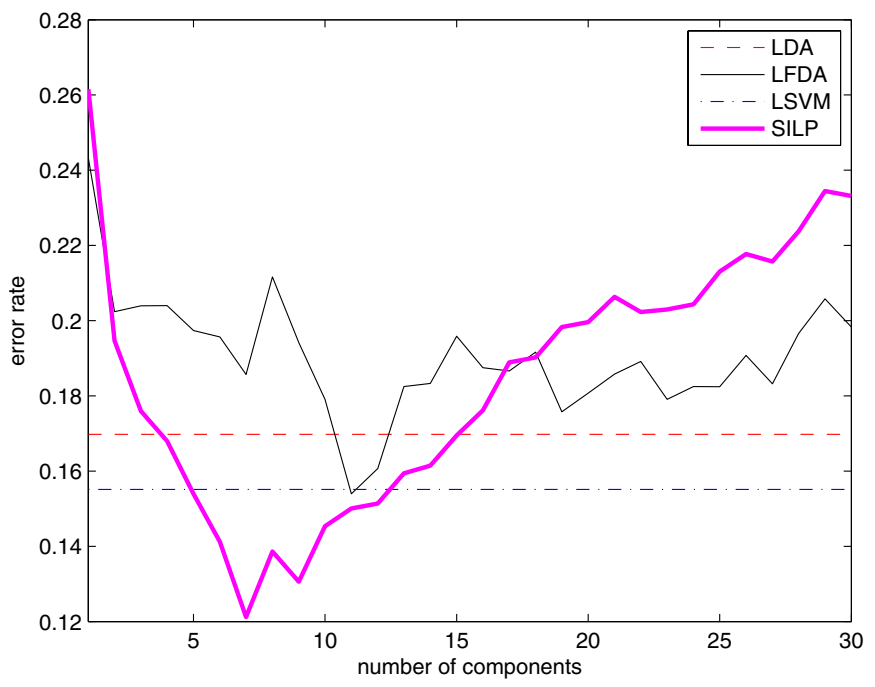

Fig. 5. Nearest neighbor classification error rates with different number of discriminative components used

least error rate $15.37 \%$. SILP is more promising in the sense that the error rate keeps decreasing with its first seven components, attaining the least classification error rate $12.29 \%$.

\section{Conclusions}

In this paper, we have incorporated the information theory into the Locality Preserving Projection and developed a new dimensionality reduction technique named Informative Laplacian Projection. Our method defines the neighborhood of a data point with its density considered. The resulting normalization factor enables the projection to encode patterns with high fidelity in sparse data areas. The proposed algorithm has been extended for extracting relevant components in supervised learning problems. The advantages of the new method have been demonstrated by empirical results on facial images.

The approach described in this paper sheds light on discovering statistical patterns for non-uniform distributions. The normalization technique may be applied to other graph-based data analysis algorithms. Yet, the challenging work is still ongoing. Adaptive neighborhood functions could be defined using advanced Bayesian learning, as spherical Gaussian kernels calculated in the input space might not work well for all kinds of data manifolds. Moreover, the transformation matrix learned by the LPP algorithm is not necessarily orthogonal. One could employ the orthogonalization techniques in [10] to enforce this constraint. Furthermore, the linear projection methods are readily extended to their nonlinear version by using the kernel technique (see e.g. 9]). 


\section{References}

1. Tenenbaum, J.B., de Silva, V., Langford, J.C.: A global geometric framework for nonlinear dimensionality reduction. Science. Science 290(5500), 2319-2323 (2000)

2. Roweis, S.T., Saul, L.K.: Nonlinear dimensionality reduction by locally linear embedding. Science 290(5500), 2323-2326 (2000)

3. Belkin, M., Niyogi, P.: Laplacian eigenmaps for dimensionality reduction and data representation. Neural Computation 15, 1373-1396 (2003)

4. Shi, J., Malik, J.: Normalized cuts and image segmentation. IEEE Transactions on Pattern Analysis and Machine Intelligence 22(8), 888-905 (2000)

5. He, X., Yan, S., Hu, Y., Niyogi, P., Zhang, H.J.: Face recognition using Laplacianfaces. IEEE Transactions on Pattern Analysis And Machine Intelligence 27, 328-340 (2005)

6. Donoho, D.L., Grimes, C.: Hessian eigenmaps: Locally linear embedding techniques for high-dimensional data. Proceedings of the National Academy of Sciences 100, 5591-5596 (2003)

7. Zhang, Z., Zha, H.: Principal manifolds and nonlinear dimensionality reduction via tangent space alignment. SIAM Journal on Scientific Computing 26(1), 318-338 (2005)

8. Belkin, M., Niyogi, P.: Laplacian eigenmaps and spectral techniques for embedding and clustering. In: Advances in Neural Information Processing Systems, vol. 14, pp. 585-591 (2002)

9. Cai, D., He, X., Zhou, K., Han, J., Bao, H.: Locality sensitive discriminant analysis. In: Proceedings of the 20th International Joint Conference on Artificial Intelligence, Hyderabad, India, January 2007, pp. 708-713 (2007)

10. Cai, D., He, X., Han, J., Zhang, H.J.: Orthogonal laplacianfaces for face recognition. IEEE Transactions on Image Processing 15(11), 3608-3614 (2006)

11. Saul, L.K., Roweis, S.: Think globally, fit locally: Unsupervised learning of low dimensional manifolds. Journal of Machine Learning Research 4, 119-155 (2003)

12. Fisher, R.A.: The use of multiple measurements in taxonomic problems. Annals of Eugenics 7, 179-188 (1963)

13. Phillips, P.J., Moon, H., Rizvi, S.A., Rauss, P.J.: The FERET evaluation methodology for face recognition algorithms. IEEE Trans. Pattern Analysis and Machine Intelligence 22, 1090-1104 (2000)

14. Newman, M.E.J.: Finding community structure in networks using the eigenvectors of matrices. Phys. Rev. 74(036104) (2006)

15. Flynn, P.J., Bowyer, K.W., Phillips, P.J.: Assessment of time dependency in face recognition: An initial study. In: Audio- and Video-Based Biometric Person Authentication, pp. 44-51 (2003)

16. Cristianini, N., Shawe-Taylor, J.: An Introduction to Support Vector Machines. Cambridge University Press, Cambridge (2000) 